\title{
Identification of critical genes to predict recurrence and death in colon cancer: integrating gene expression and bioinformatics analysis
}

Xuan Long ${ }^{1,2}$, Zhigang Deng ${ }^{2}$, Guoqiang $\mathrm{Li}^{2}$ and Ziwei Wang ${ }^{1 *}$

\begin{abstract}
Background: The purpose of this study was to screen the critical genes for future diagnosis and treatment of colon cancer by bioinformatics method.

Methods: In this study, we used bioinformatics approaches to identify gene alteration that contribute to colon cancer progression via analysis of TCGA RNA sequencing data and other publicly GEO microarray data. The Random forest survival model was used to screen gene sets related to the prognosis in DEGs. Gene ontology and KEGG pathway enrichment analysis were performed to determine the potential function of DEGs.

Results: We identified versican (VCAN), a member of the aggrecan/versican proteoglycan family, as a key regulator in human colon cancer development and progression involved in cell adhesion, proliferation, migration and angiogenesis and plays a central role in tissue morphogenesis and maintenance. Interestingly, we found that VCAN is highly over-expressed in colon cancer and increased expression of VCAN was associated with the progression of colon cancer. High VCAN levels also predict shorter overall survival of colon cancer patients. Furthermore, in vitro assays of silencing VCAN inhibit HCT116 cell proliferation and invasion.

Conclusions: These data demonstrated VCAN were associated with tumorigenesis and may be as biomarker for identification of the pathological grade of colon cancer.
\end{abstract}

Keywords: Biomarker, Colon cancer, VCAN

\section{Background}

More than 1.2 million patients are diagnosed with colon cancer every year, and more than 600,000 die from the disease [1-4]. Incidence strongly varies globally and is closely linked to elements of a so-called western lifestyle. Incidence is higher in men than women and strongly increases with age; median age at diagnosis is about 70 years in developed countries [5-8]. Despite strong hereditary components, most cases of colon cancer are sporadic and develop slowly over several years through

\footnotetext{
*Correspondence: zwwangcamu@163.com

${ }^{1}$ Department of Gastroenterological Surgery, The First Affiliated Hospital of Chongqing Medical University, Chongqing Medical University, 1 \# Youyi Road, Chongqing 400016, People's Republic of China

Full list of author information is available at the end of the article
}

the adenoma-carcinoma sequence. The cornerstones of therapy are surgery, neoadjuvant radiotherapy (for patients with rectal cancer), and adjuvant chemotherapy (for patients with stage III/IV and high-risk stage II colon cancer) [9-11]. 5-year relative survival ranges from greater than $90 \%$ in patients with stage I disease to slightly greater than $10 \%$ in patients with stage IV disease. Screening has been shown to reduce colon cancer incidence and mortality, but organised screening programmes are still to be implemented in most countries [12-14].

Using high-throughput technology to analyze gene expression data can solve the current problem mentioned above. The gene expression profile of colon cancer had analyzed by microarray technique indicated many genes 
was a key factor affecting the disease progress $[15,16]$. But few differentially expressed genes (DEGs) have been reported. Microarray technology combining with bioinformatics analysis makes it possible to comprehensively analyze the DEGs in mRNA expression level in the development and progression of colon cancer.

We identified versican (VCAN), a member of the aggrecan/versican proteoglycan family, as a key regulator in human colon cancer development and progression involved in cell adhesion, proliferation, migration and angiogenesis and plays a central role in tissue morphogenesis and maintenance. This gene is a member of the aggrecan/versican proteoglycan family. The protein encoded is a large chondroitin sulfate proteoglycan and is a major component of the extracellular matrix $[17,18]$. This protein is involved in cell adhesion, proliferation, migration and angiogenesis and plays a central role in tissue morphogenesis and maintenance. Mutations in this gene are the cause of Wagner syndrome type 1 [19]. Multiple transcript variants encoding different isoforms have been found for this gene.

\section{Materials and methods Data sources analysis}

Raw gene expression data and clinical profile were downloaded from The Cancer Genome Atlas Data Portal (https://gdc-portal.nci.nih.gov/search/s) and Gene Expression Omnibus dataset in National Center for Biotechnology Information (https://www.ncbi.nlm.nih.gov/ geo/). "Limma" $\mathrm{R}$ package was used to identify DEGs between pediatric ependymoma samples and control samples. After running the "limma" package we got a matrix with 54,675 rows and 6 columns. As we knew, the $\log$ FC column gave the value of the contrast. Column $P$ value was the associated $P$-value and adj. $P$-value was the $P$-value adjusted for multiple testing. In this analysis $P$-value $<0.05$ and $|\operatorname{logFC}|>2$ were regarded as the cutoff criterion for EDGs.

\section{GO functional and pathway enrichment analysis}

One of the main uses of GO is to perform enrichment analysis on gene sets. For example, given a set of genes that were up regulated under certain conditions, the enrichment analysis would find which GO terms were over-expressed or down-expressed using annotation for that gene set. KEGG pathway was a collection of manually drawn pathway maps representing our knowledge on the molecular interaction and reaction network for cellular processes, human diseases and so on. Because we had got the DEGs annotation by R package from the latest version of bioconductor (library "affy", "limma" and "hgu133plus2.db"). So we could do GO and KEGG analysis in search tool for the retrieval of interacting genes (STRING) database version 10.0 on line.

\section{Immunohistochemical staining}

For immunohistochemistry, slides were routinely deparaffinized and rehydrated, and then were subjected to heat-induced epitope retrieval in $0.01 \mathrm{mM}$ citrate buffer ( $\mathrm{pH}$ 6.0). Endogenous peroxidase activity was blocked for $10 \mathrm{~min}$ in $3 \%$ hydrogen peroxide and methanol. The slides were then incubated with rabbit Anti-VCAN polyclonal antibody (1:200; ab19345; Abcam Technology) at $4{ }^{\circ} \mathrm{C}$ overnight. Sections were then stained with DAB (Maixin. Bio, Fuzhou, China) for $5 \mathrm{~min}$. Specific VCAN ISH signal was identified as brown, punctate dots and expression level was scored as Image-Pro plus 6.0 software. The intensity of staining was scored as 0 (negative), 1 (weakly positive), 2 (moderately positive), and 3 (strongly positive). According to the percentage of the positive staining area, the extent of staining was scored as $0(0-10 \%), 1(11 \%-30 \%)$, $2(31 \%-50 \%), 3(51 \%-70 \%)$, and $4(71 \%-100 \%)$. The final staining scores (ranging from 0 to 7 ) of VCAN expression were divided into two groups:high expression groups (scores $\geq 3$ ) and low expression groups (scores $<3$ ).

\section{Cell proliferation and migration assay}

The small interference RNA (siRNA) was designed by Sangon biotech. The siRNAs were transfected into HCT116 cells using Lipofectamine ${ }^{\circledR}$ RNAiMAX (Invitrogen) according to the manufacturer's instructions. Colony formation assays were performed to detect HCT-116 cells cloning Capability after HCT-116 cells transfected with si-VCAN or si-NC. During migration assay, endothelial cells are placed on the upper layer of a cell permeable membrane and a solution containing the test agent is placed below the cell permeable membrane. Following an incubation period $(18 \mathrm{~h})$, the cells that have migrated through the membrane are stained and counted.

\section{Statistical analysis}

For microarray analysis, differentially expressed genes were confirmed using a $P$-value threshold and FDR analysis. The threshold of truly significant miRNA was taken to be $P$ value $<0.05$ and FDR value $<0.05$. The statistical analysis performed with the software of SPSS version 18.0 for Windows. All the data were expressed as mean \pm SD. The statistical significance was evaluated by ANOVA or two-tailed $t$ test, and the results were considered significant at a $P$ value $<0.05$. 


\section{Results}

Identification of differently expressed genes (DEGs) in human colon cancer

To identify DEGs that are played key role in colon tumorigenesis, we used an integrative analysis of TCGA colon adenocarcinoma (TCGA-COAD) and RNA-seq data and colon cancer gene expression data includinging GSE63624, and GSE77167 the publicly available GEO databases. We identified 175 genes deregulated in the TCGA data, 77 in GSE63624 datasets, and 57 in GSE77167 datasets under the condition of " $\mathrm{Q}<0.001$ and fold change $>4$ ". Total these DEGs are shown clustered in Fig. 1a, then we founded only five genes consistently up-regulated and four down-regulated in all datasets (Fig. 1b).

\section{GO functional and pathway enrichment analysis}

To determine significantly DEGs in human colon cancer, gene ontology (GO) and pathway enrichment analysis were performed. We showed that the up/downregulated DEGs were significantly enriched in wound healing involved in inflammatory response, positive regulation of phosphatase activity, cellular response to erythropoietin, negative regulation of skeletal muscle

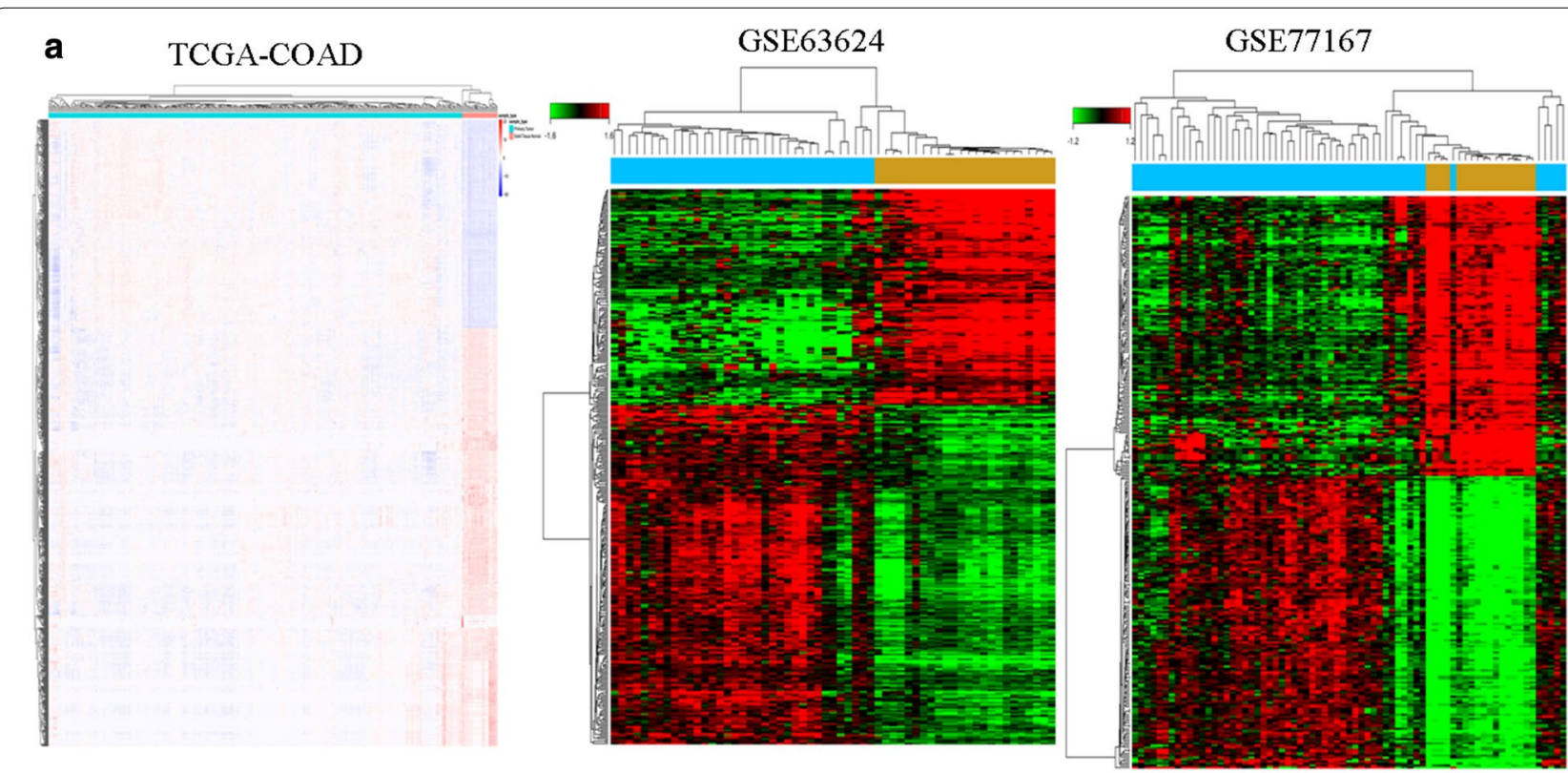

b Over-expressed

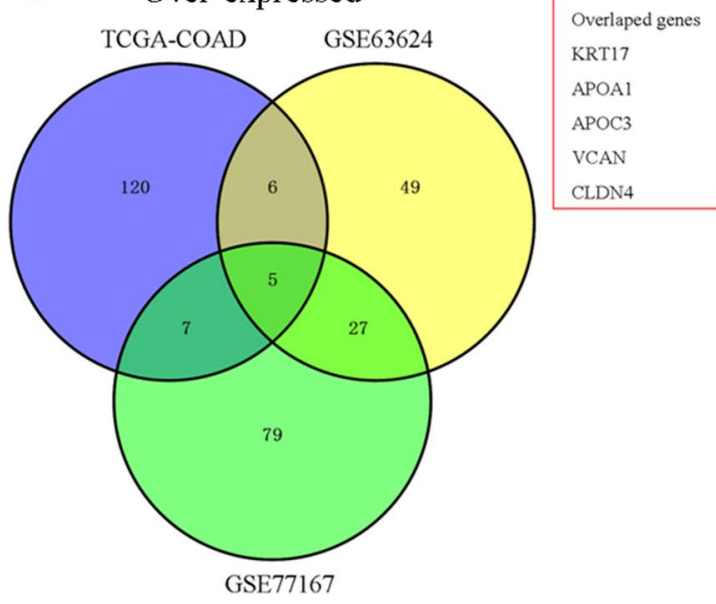

Under-expressed

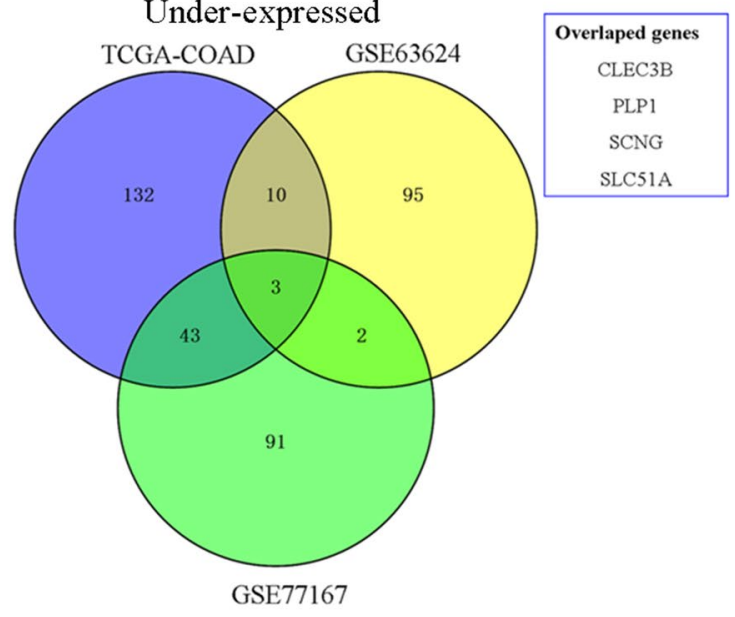

Fig. 1 Identification of differently expressed genes (DEGs) in human colon cancer (a) hierarchical clustering analysis of genes that were differentially expressed (fold change $>4 ; P<0.05$ ) in colon cancer and normal tissues (b) overlap of misregulated genes in TCGA data and GEO datasets 
wound healing involved in inflammatory response positive regulation of $\mathrm{p} 38 \mathrm{MAPK}$ cascade

UDP-glucose metabolic process cellular biogenic amine metabolic process

response to lipid hydroperoxide positive regulation of phosphatase activity free ubiquitin chain polymerization cellular response to erythropoietin establishment of planar polarity of embryonic epithelium negative regulation of skeletal muscle cell differentiation

trehalose biosynthetic process purine nucleobase biosynthetic process GMP biosynthetic process mesenchyme development

female meiosis re-entry into mitotic cell cycle

UDP-glucuronate biosynthetic process negative regulation of planar cell polarity pathway involved in axis elongation mitotic spindle checkpoint spindle assembly checkpoint
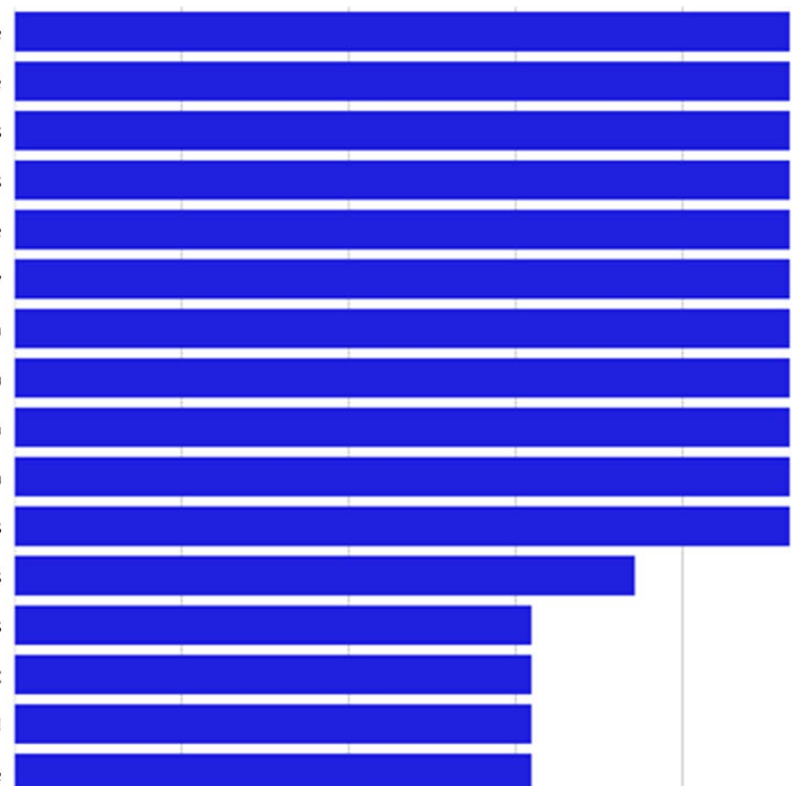

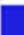

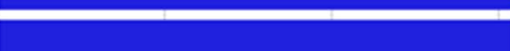

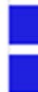

0

10

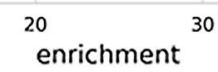
small molecule metabolic process
transmembrane transport mitotic cell cycle signal transduction$$
\text { cell division }
$$ positive regulation of cell proliferation negative regulation of cell proliferation muscle contraction blood coagulation carbohydrate metabolic process one.carbon metabolic process lipid metabolic process xenobiotic metabolic process rRNA processing cell proliferation negative regulation of transcription from RNA polymerase II promoter nucleobase-containing small molecule metabolic process M phase of mitotic cell cycle

$$
\text { mitosis }
$$

ion transport
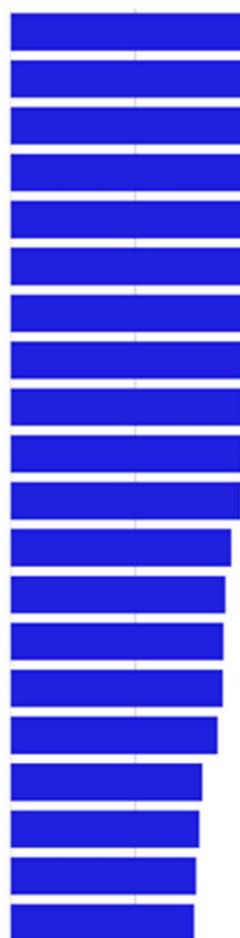

$0 \quad 5$

10

15

20

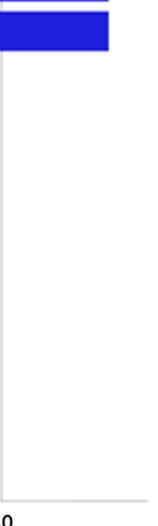

- $\log 10$ (Pvalue)

Fig. 2 GO functional and pathway enrichment analysis of differently expressed genes (DEGs) in human colon cancer 

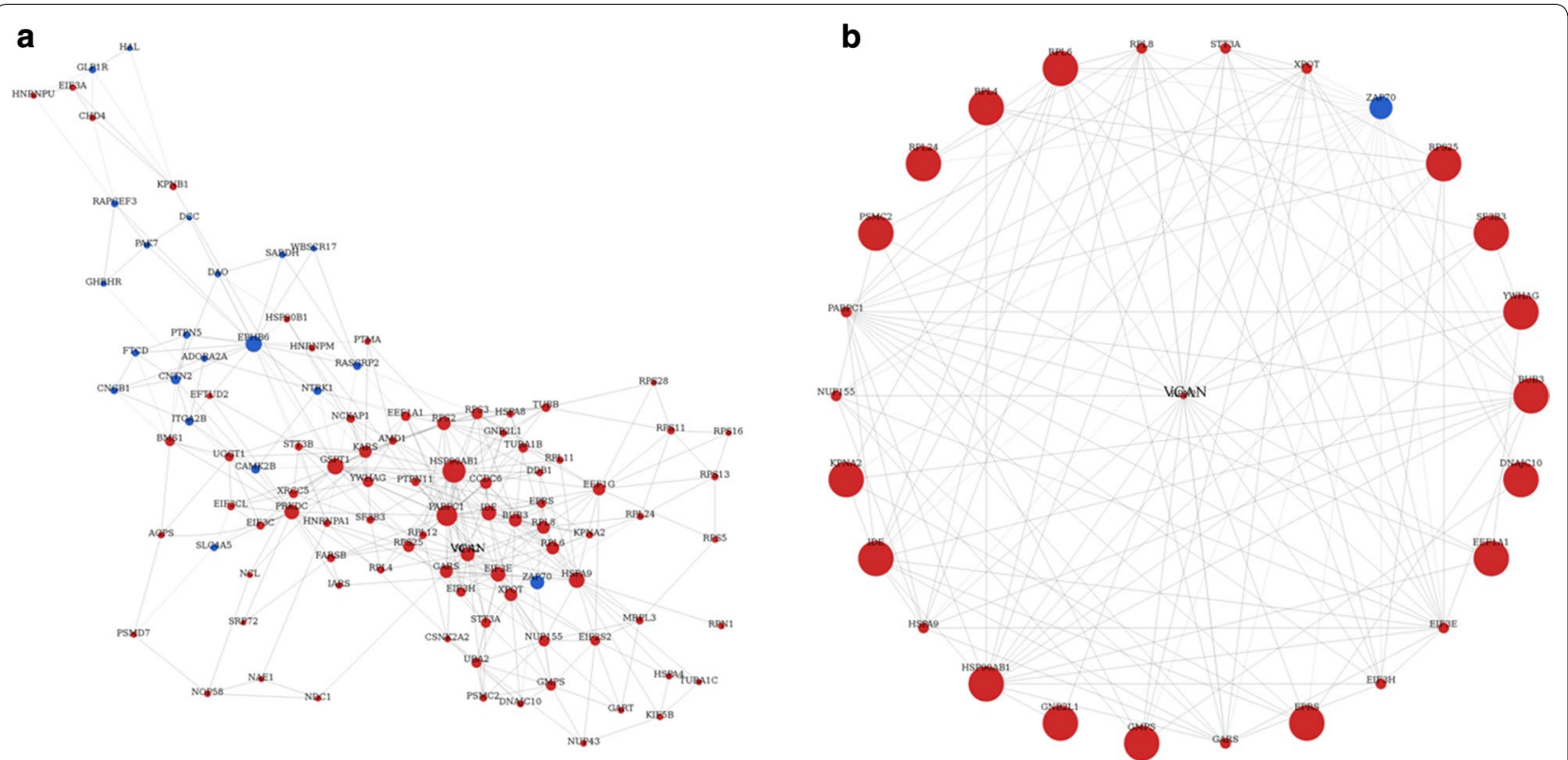

Fig. 3 mRNA-mRNA co-expression network. a The differential genes were selected as candidate genes as function of IDC by constructing a gene co-expression network with k-core algorithm. b VCAN was the key gene in the gene network. Node size represents the degree centrality

cell differentiation, mitotic cell cycle, and cell division. Important genes and pathways involved in this process are shown in Fig. 2.

\section{Co-expression gene-network analysis and candidate biomarker identification}

To determine which gene or genes may play a pivotal role in the development of human colon cancer, we construct a gene-gene co-expression network. This co-expression network indicated HSP90AB1, VCAN, CLDN2, EPHB6, EIF3E, GSPT1, PRKDC, RPS2, GARS etc. play a key role in the progression of colon cancer (Fig. 3a). We selected VCAN over-expression genes because may be useful for early diagnosis biomarkers or therapeutic targets (Fig. 3b). We next further analysis the expression of in Colon adenocarcinoma (COAD), Adrenocortical carcinoma (ACC), Bladder Urothelial Carcinoma (BLCA), Breast invasive carcinoma (BRCA), Cervical squamous cell carcinoma and endocervical adenocarcinoma (CESC) using TCGA sequencing data and founded that is specifically upregulated in colon cancer (Fig. 4a).

\section{VCAN expression is elevated in primary human colon cancer}

To investigate the correlation between VCAN expression and survival of colon cancer patient, we conducted a Kaplan-Meier analysis with TCGA samples dichotomized into 2 groups with expression levels less than or equal to median and levels more than median of expression. We fouded that patients in the high-risk group had significantly shorter median DFS than those in the low-risk group (Fig. 4b, c). qRT-PCR analysis were performed to detected the expression profile of VCAN in a panel of colon cancer cDNA arrays including 30 patients with colon cancer and 30 healthy controls. Result showed VCAN was significantly up-regulated at the mRNA level in colon cancer samples compared with normal colon tissues (Fig. 4d). We also founded that the expression level of VCAN mRNA was positively correlated with clinical stage $(P<0.05)$, (Fig. $4 \mathrm{e})$.

\section{VCAN identified as a potential novel prognostic biomarker} Immunohistochemical staining analysis in 100 human colon cancers and matched 60 adjacent tissue microarrays showed that VCAN was significantly overexpressed in colon cancer samples compared with adjacent tissue (Fig. 5a). Kaplan-Meier survival analysis showed that the overall survival and progression-free survival rates over 3 years for the high VCAN group were lower than those in the low VCAN group (Fig. 5b). Interestingly, we founded that VCAN expression levels significantly correlated with tumor size $(P=0.012)$ and clinical stage $(P=0.015)$ in colon cancer, but not 

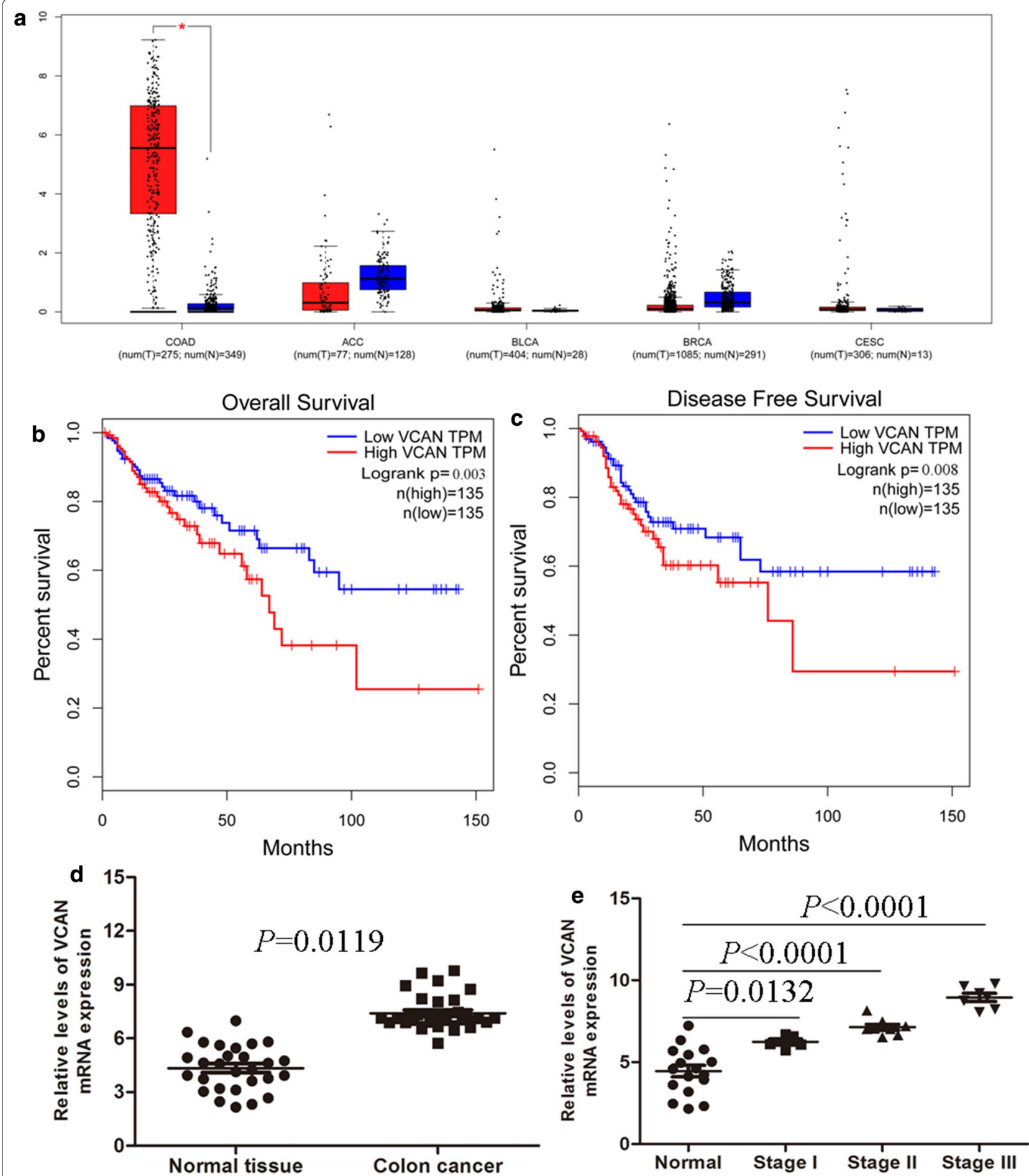

Fig. 4 VCAN expression is elevated in primary human colon cancer. a Analyses of VCAN expression levels in colon adenocarcinoma (COAD), adrenocortical carcinoma (ACC), bladder urothelial carcinoma (BLCA), breast invasive carcinoma (BRCA), cervical squamous cell carcinoma and endocervical adenocarcinoma (CESC) using TCGA sequencing data. b, c Kaplan-Meier survival analysis of overall survival and disease-free survival in colon cancer patients ( $P<0.001$ for both overall survival and disease-free survival) based on VCAN expression using TCGA sequencing data. $\mathbf{d}$ VCAN expression was analyzed by qRT-PCR in colon cancer and adjacent nontumor tissues $(n=30)$. e The relationship between VCAN mRNA expression levels and clinic-pathologic parameters 

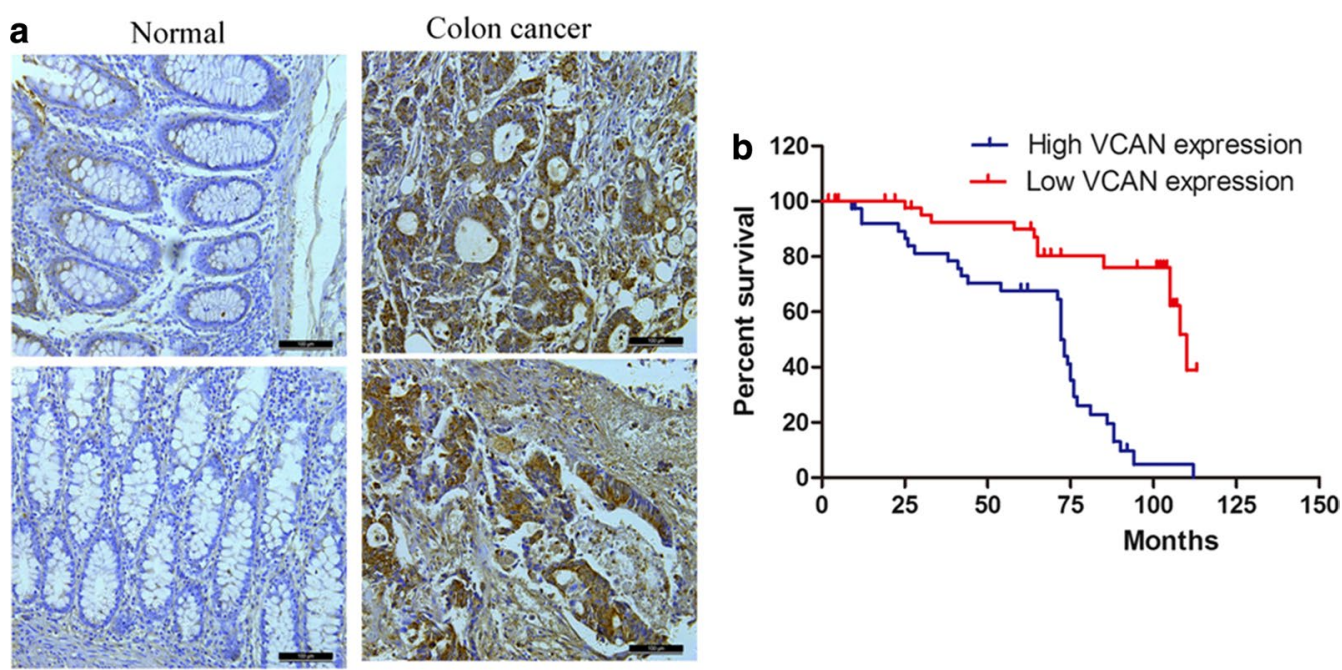

Fig. 5 Expression of VCAN in colon cancer patient specimens. a Expression of VCAN in primary colon cancer and matched normal tissue $(\times 100$ or $\times 400$ ). b Kaplan-Meier plots of VCAN expression in 37 cases of colon cancer patients. Overall survival rate was performed by log-rank test. (Immunoreactivity scores $<4$ was ascribed to be low VCAN expression, immunoreactivity scores $\geq 4$ was ascribed to be high VCAN expression)

Table 1 Correlation of VCAN protein expression with clinicopathological data (Fisher's exact test)

\begin{tabular}{lcccc}
\hline Pathological variables & Sample & \multicolumn{2}{l}{$\begin{array}{l}\text { VCAN IHC staining } \\
\text { (\%) }\end{array}$} & P value \\
\cline { 3 - 4 } & & Negative & Positive & \\
\hline Normal tissues & 60 & $50(83.3)$ & $10(16.7)$ & $0.016^{*}$ \\
Primary tumours & 100 & $20(20.0)$ & $80(80.0)$ & \\
Stage & & & & $0.015^{*}$ \\
I+ II & 70 & $14(20.0)$ & $56(80.0)$ & \\
III+ IV & 30 & $9(30.0)$ & $21(70.0)$ & \\
Lymph node status & & & & 0.521 \\
pN0 & 72 & $14(19.4)$ & $58(80.6)$ & \\
pN1+ & 28 & $8(28.5)$ & $20(71.5)$ & \\
Grade & & & & 0.241 \\
1 & 32 & $6(18.7)$ & $26(91.3)$ & \\
2 & 40 & $10(25.0)$ & $30(75.0)$ & \\
3 & 28 & $9(32.1)$ & $19(67.9)$ & \\
Tumour size & & & & 0.012 \\
T1-3 & 56 & $12(21.4)$ & $44(78.6)$ & \\
T4 & 44 & $10(22.7)$ & $34(77.3)$ & \\
\hline
\end{tabular}

${ }^{*} P$ value $<0.05$ was consider significant

associated with other factors including pathological grading and lymph node status as shown in Table 1.
Silencing of VCAN inhibits HCT-116 cell colony formation and migration

To determine the function of VCAN in regulating human colon cancer cell phenotype, we next performed knockdown of VCAN in HCT-116 cell line that with higher VCAN expression using small interfering RNA. Quantitative RT-PCR and Western blot analysis to quantitatively measure the effect of VCAN knockdown. Results that the VCAN expression was significantly decreased at both mRNA and protein levels in HCT116 cell lines (Fig. 6a, b). Transwell migration assays showed that knockdown of VCAN dramatically decreased cell migration (Fig. 6c, d). Furthermore, Colony formation assays showed that knockdown of VCAN inhibited cell proliferation in vitro.

\section{Discussion}

Treatments used for colon cancer may include some combination of surgery, radiation therapy, chemotherapy and targeted therapy [20-22]. Cancers that are confined within the wall of the colon may be curable with surgery while cancer that has spread widely are usually not curable, with management being directed towards improving quality of life and symptoms [23]. Five year survival rates in the United States are around 65\%. This, however, depends on how advanced the cancer is, whether or not all the cancer can be removed with surgery, and the person's overall health. Globally, colon cancer is the third most common type of cancer making up about $10 \%$ of all cases. In 2012, there were 1.4 


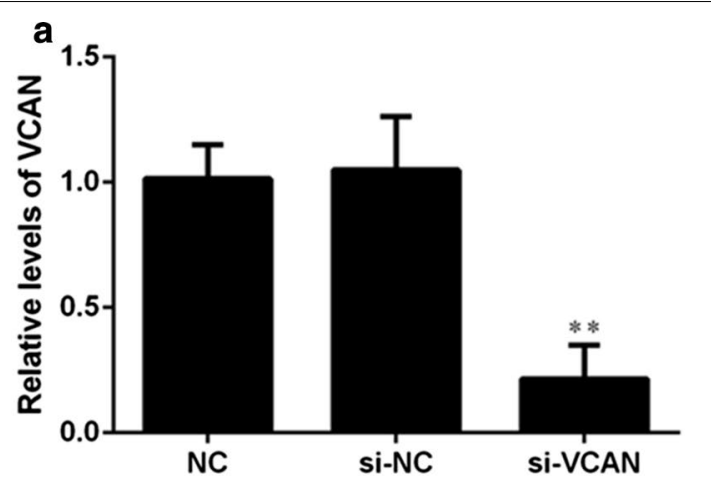

b
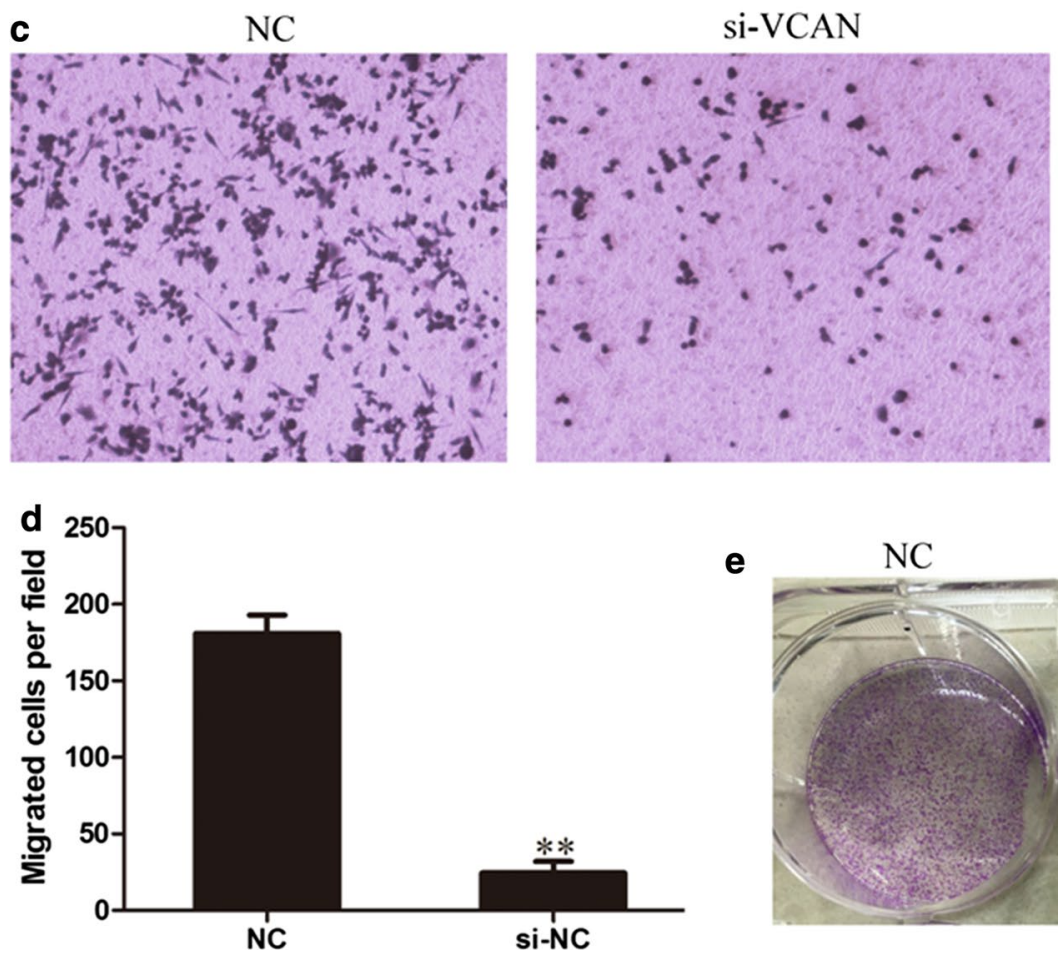

e

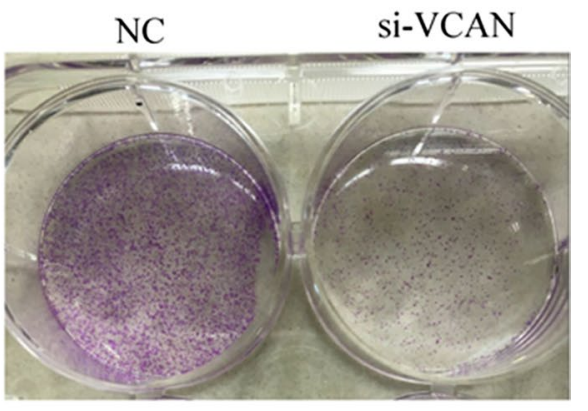

Fig. 6 Knock-down of VCAN inhibits HCT-116 cell colony formation and migration (a, b) small interfering RNA (siRNA)-mediated knockdown ofVCAN. HCT116 cells were transfected with negative control siRNA (NC) and siRNA against VCAN (si-VCAN). After transfection, Expression of VCAN was determined by qRT-PCR and immunoblot analysis. HCT116 cells were transiently transfected with negative control siRNA and siRNA against VCAN, and then subjected to $(\mathbf{c}, \mathbf{d})$ transwell migration assay, and e colony formation, respectively. ${ }^{* *} p<0.01$

million new cases and 694,000 deaths from the disease $[6,24]$.

Previous studies demonstrate VCAN is involved in cell adhesion, proliferation, migration and angiogenesis and plays a central role in tissue morphogenesis and maintenance. Zhao et al. reported miR-135a-5p could affect the proliferation, invasion and migration of thyroid carcinoma cells by targeting VCAN [18]. Sathyan et al. reported Versican plays an important role in extracellular matrix assembly and plays a major role in the pathogenesis of IA [17]. The linkage studies also indicated VCAN as a putative candidate gene for
IA in the 5q22-31 region. Chida et al. reported VCAN protein was detected exclusively in cancer stroma by immunohistochemistry, demonstrating a stepwise increase of stromal VCAN from normal tissues through stage 0 to stage IV tumors [25].

Our data demonstra that the expression level of VCAN mRNA was positively correlated with pathologic grade, clinical stage, VCAN were significantly over-expressed in metastasis samples compared with primary tumors. Immunohistochemical staining analysis in 100 human colon cancers and matched adjacent tissue microarray showed that VCAN was significantly over-expressed in 
colon cancer samples compared with adjacent tissue. A loss-of-function study revealed that colony formation assays showed that knockdown of VCAN inhibited cell proliferation in vitro. Transwell migration assays showed that knockdown of VCAN dramatically decreased cell migration.

\section{Conclusions}

In conclusion, we demonstrated for the first time that VCAN is over-expressed in colorectal cancer and VCAN promotes colorectal cancer cell growth in vitro. These data suggest VCAN might serve as a potential target in the diagnosis and/or treatment in colorectal cancer.

\begin{abstract}
Abbreviations
COAD: colon adenocarcinoma; ACC: adrenocortical carcinoma; BLCA: bladder urothelial carcinoma; BRCA: breast invasive carcinoma; CESC: cervical squamous cell carcinoma and endocervical adenocarcinoma; qRT-PCR: quantitative reverse transcription polymerase chain reaction; PBS: phosphate buffered saline; TCGA: The Cancer Genome Atlas; GEO: gene expression omnibus.
\end{abstract}

\section{Authors' contributions}

We thanked XL for clinical information collection. Performed the experiments and collected data: XL, ZW. Analyzed the data: ZD. Conceived and designed the experiments: GL. Contributed reagents/materials: ZW. Wrote the paper: XL, ZW. Revised the paper: ZW. All authors read and approved the final manuscript.

\section{Author details}

1 Department of Gastroenterological Surgery, The First Affiliated Hospital of Chongqing Medical University, Chongqing Medical University, 1 \# Youyi Road, Chongqing 400016, People's Republic of China. ${ }^{2}$ Department of General Surgery, Mianyang Central Hospital, Mianyang 621000, Sichuan, People's Republic of China.

\section{Acknowledgements}

We thanked Rong Jiang of Chongqing Medical University for clinical information collection.

\section{Competing interests}

The authors declare that they have no competing interests.

\section{Availability of data and materials}

The datasets used and/or analysed during the current study are available from the corresponding author on reasonable request.

\section{Availability of data and materials}

The datasets used and/or analysed during the current study are available from the corresponding author on reasonable request.

\section{Consent for publication}

The manuscript did not contain any individual person's data in any form.

\section{Ethics approval and consent to participate}

The study has been approved by the ethical committee of Chongqing Medical University.

\section{Funding}

This work was supported by National Natural Science Foundation of China (No. 81272753).

\section{Publisher's Note}

Springer Nature remains neutral with regard to jurisdictional claims in published maps and institutional affiliations.

Received: 28 May 2018 Accepted: 10 September 2018

Published online: 17 September 2018

\section{References}

1. O'Keefe SJ. Diet, microorganisms and their metabolites, and colon cancer. Nat Rev Gastroenterol Hepatol. 2016;13(12):691-706.

2. Irrazabal T, Belcheva A, Girardin SE, Martin A, Philpott DJ. The multifaceted role of the intestinal microbiota in colon cancer. Mol Cell. 2014;54(2):309-20.

3. Segal NH, Saltz LB. Evolving treatment of advanced colon cancer. Annu Rev Med. 2009;60:207-19.

4. Rustgi AK. The genetics of hereditary colon cancer. Genes Dev. 2007:21(20):2525-38.

5. Tauriello DVF, Palomo-Ponce S, Stork D, Berenguer-Llergo A, BadiaRamentol J, Iglesias M, Sevillano M, Ibiza S, Canellas A, Hernando-Momblona $X$, et al. TGFbeta drives immune evasion in genetically reconstituted colon cancer metastasis. Nature. 2018;554(7693):538-43.

6. Chung DC. Genetic testing and early onset colon cancer. Gastroenterology. 2018;154(4):788-9.

7. Seidel DV, Azcarate-Peril MA, Chapkin RS, Turner ND. Shaping functiona gut microbiota using dietary bioactives to reduce colon cancer risk. Semin Cancer Biol. 2017;46:191-204.

8. Viguier J, Morere JF, Brignoli-Guibaudet L, Lhomel C, Couraud S, Eisinger F. Colon cancer screening programs: impact of an organized screening strategy assessed by the EDIFICE surveys. Current oncology reports. 2018;20(Suppl 1):16.

9. Wu C. Systemic therapy for colon cancer. Surg Oncol Clin N Am. 2018;27(2):235-42.

10. Yu L, Zhou Y, Yang Y, Lu F, Fan Y. Efficacy and safety of compound kushen injection on patients with advanced colon cancer: a meta-analysis of randomized controlled trials. eCAM. 2017;2017:7102514.

11. Gkekas I, Novotny J, Pecen L, Strigard K, Palmqvist R, Gunnarsson U. Microsatellite instability as a prognostic factor in stage II colon cancer patients, a meta-analysis of published literature. Anticancer Res. 2017:37(12):6563-74.

12. Merlano MC, Granetto C, Fea E, Ricci V, Garrone O. Heterogeneity of colon cancer: from bench to bedside. ESMO open. 2017;2(3):e000218.

13. Franklin BR, McNally MP. Laparoscopy for colon cancer. Clin Colon Rectal Surg. 2017;30(2):99-103.

14. Conde J, Oliva N, Zhang Y, Artzi N. Local triple-combination therapy results in tumour regression and prevents recurrence in a colon cancer model. Nat Mater. 2016;15(10):1128-38.

15. Staal FJ, van der Burg M, Wessels LF, Barendregt BH, Baert MR, van den Burg CM, van Huffel C, Langerak AW, van der Velden VH, Reinders MJ, et al. DNA microarrays for comparison of gene expression profiles between diagnosis and relapse in precursor-B acute lymphoblastic leukemia: choice of technique and purification influence the identification of potential diagnostic markers. Leukemia. 2003;17(7):1324-32.

16. Centeno BA, Enkemann SA, Coppola D, Huntsman S, Bloom G, Yeatman TJ. Classification of human tumors using gene expression profiles obtained after microarray analysis of fine-needle aspiration biopsy samples. Cancer. 2005;105(2):101-9.

17. Sathyan S, Koshy LV, Balan S, Easwer HV, Premkumar S, Nair S, Bhattacharya RN, Alapatt JP, Banerjee M. Association of Versican (VCAN) gene polymorphisms rs251124 and rs2287926 (G428D), with intracranial aneurysm. Meta Gene. 2014;2:651-60.

18. Zhao X, Sun Z, Li H, Jiang F, Zhou J, Zhang L. MiR-135a-5p modulates biological functions of thyroid carcinoma cells via targeting VCAN 3'-UTR. Cancer Biomark. 2017:20(2):207-16.

19. Burin-des-Roziers C, Rothschild PR, Layet V, Chen JM, Ghiotti T, Leroux C, Cremers FP, Brezin AP, Valleix S. Deletions overlapping VCAN exon 8 are new molecular defects for wagner disease. Hum Mutat. 2017;38(1):43-7. 
20. Venook AP. Advances in adjuvant therapy for colon cancer: $p$ value or practical value. Journal of clinical oncology. 2018. https://doi. org/10.1200/JCO.2018.77.8423.

21. Andre T, Vernerey D, Mineur L, Bennouna J, Desrame J, Faroux R, Fratte $S$, Hug de Larauze M, Paget-Bailly S, Chibaudel B, et al. 3 versus 6 months of oxaliplatin-based adjuvant chemotherapy for patients with stage III colon cancer: disease-free survival results from a randomized, open-label, international duration evaluation of adjuvant (IDEA) France, phase III trial. Journal of clinical oncology. 2018:36:1469-77.

22. Ilson DH. Adjuvant therapy in colon cancer: less is more. Lancet Oncol. 2018;19(4):442-3.
23. Park H, Chen B, Ciorba MA. Progress in PD-1-based Immunotherapy: new mechanistic insight may provide expanded hope for application to colon and gastrointestinal cancers. Gastroenterology. 2017;153(4):1162-3.

24. Fadelu T, Zhang S, Niedzwiecki D, Ye X, Saltz LB, Mayer RJ, Mowat RB, Whittom $R$, Hantel $A$, Benson AB, et al. Nut consumption and survival in patients with stage III colon cancer: results from CALGB 89803 (Alliance). J Clin Oncol. 2018:36(11):1112-20.

25. Chida S, Okayama H, Noda M, Saito K, Nakajima T, Aoto K, Hayase S, Momma T, Ohki S, Kono K, et al. Stromal VCAN expression as a potential prognostic biomarker for disease recurrence in stage II-III colon cancer. Carcinogenesis. 2016;37(9):878-87.
Ready to submit your research? Choose BMC and benefit from:

- fast, convenient online submission

- thorough peer review by experienced researchers in your field

- rapid publication on acceptance

- support for research data, including large and complex data types

- gold Open Access which fosters wider collaboration and increased citations

- maximum visibility for your research: over $100 \mathrm{M}$ website views per year

At BMC, research is always in progress.

Learn more biomedcentral.com/submissions 